\title{
MICROLENSING AND DYNAMICS OF THE GALACTIC BULGE
}

the importance of being earnest

\author{
H.S.ZHAO \\ Leiden Observatory, Niels Bohrweg 2, 2333 CA, Leiden, The \\ Netherlands
}

\begin{abstract}
Microlensing ( $\mu$-lensing) towards the bulge started out as a unique technique of detecting dark objects in the Galactic disc (brown dwarfs) by measuring a rare transient brightening of one star among millions of stars in the Galactic bulge (Paczyński 1991). More than 200 events have been detected towards the Bulge since 1993 by the DUO, MACHO, OGLE etc. survey teams. But where are the lenses? Are they truly dark objects or merely faint stars? I show the results on the mass function, spatial distribution and dynamics of these observed lenses based on a dynamical model of the Galactic bar.
\end{abstract}

It is well demonstrated by the COBE/DIRBE infrared maps of the Galactic plane that the central region of the Galaxy appears systematically brighter at $l>0^{\circ}$ side than $l<0^{\circ}$, as expected from a triaxial bar pointing at $15^{\circ}-45^{\circ}$ from our line of sight. Using a dynamical model for the COBE bar (Zhao 1996), we predict that lenses in the near side of the massive bar at the center dominate those in the disc at half way to the center. Most of the lenses should be at about $3 / 4$ of our distance to the center.

Fig. 1 shows that present day mass functions proposed by Kroupa, Tout \& Gilmore (1993) is roughly consistent with the observed distribution with or without upper and lower cutoffs; models which contain more than $25 \%$ brown dwarfs are ruled out at a high confidence level. This suggests that in principle one could observe the light from the lenses since the median lens mass is $0.16 M_{\odot}$, faint stars above hydrogen burning limit $0.08 M_{\odot}$, consistent with Zhao, Rich \& Spergel (1996).

Most parameters of a lens $\left(D_{\text {lens }}, \vec{V}_{\text {lens }}, m_{\text {lens }}\right)$ are unobservable. Dynamical models are needed to simulate these parameters $\left(D_{l e n s}, \vec{V}_{l e n s}\right.$ in a Monte-Carlo fashion. Then from the observed event duration $t_{o b s}$ one can 

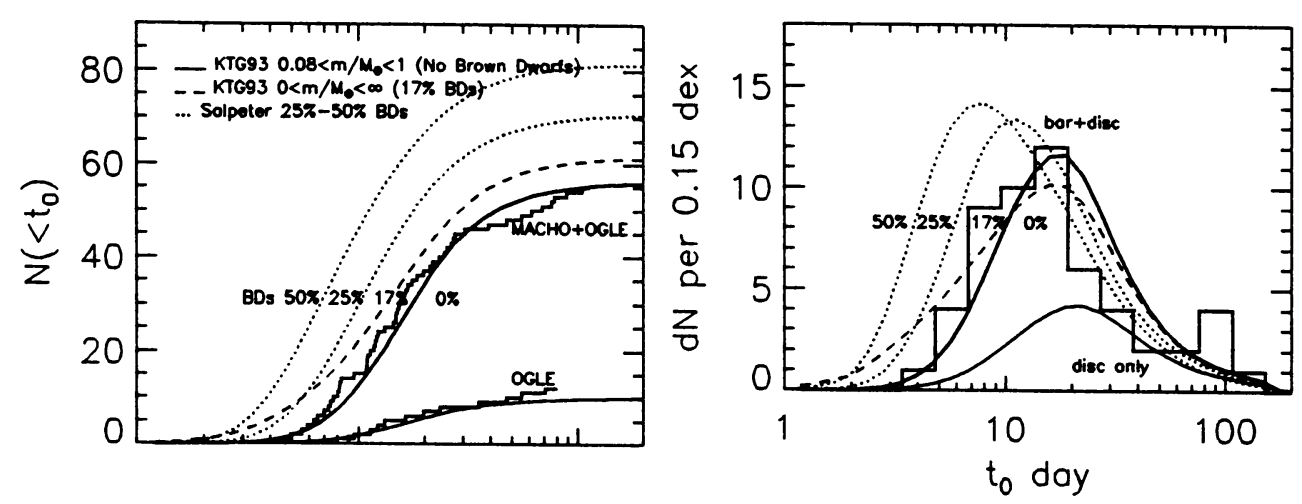

Figure 1. shows the differential and cumulative distributions of the observed events in histograms and the prediction using mass functions with different fraction of brown dwarfs. Brown dwarf fraction has to be low.

infer the lens mass function according to the relation

$$
m_{l e n s} \propto \frac{V^{2} t_{o b s}^{2}}{D} .
$$

The above equation suggests that the lens mass can be a strong function of the shape of the deprojected bulge/bar and its streaming motion. Unfortunately it is not possible to constrain the volume density of the bar uniquely from the COBE/DIRBE maps (Zhao 1997 and references therein), particularly, it is difficult to distinguish among a sequence of bar models related by a shear transformation in the line of sight direction. Thin long bars have a shallower potential than slightly barred models, and a lower velocity dispersion for the lenses and sources (Zhao \& de Zeeuw 1997), so the infer' ' lens mass has to decrease (cf. eq. 1) to fit the same observed event rate. The models we have used are self-consistent models which fit both the COBE map and gas/stellar dynamics. Such models predict that most of the lens are faint stars at the near end of the bar.

\section{References}

Kroupa P., Tout C.A. \& Gilmore G. 1993, MNRAS, 262, 545

Paczyński B., 1991, ApJ, 371, L63

Zhao H.S., 1996, MNRAS, 283, 149

Zhao H.S., 1997, MNRAS, submitted

Zhao H.S., de Zeeuw P.T. 1997, MNRAS, submitted

Zhao H.S., Rich R.M., Spergel D.N., 1996, MNRAS, 282, 175 